\title{
Control Capabilities of Myoelectric Robotic Prostheses by Hand Amputees: A Scientific Research and Market Overview
}

\author{
Manfredo Atzori* and Henning Müller \\ Information Systems Institute, University of Applied Sciences Western Switzerland (HES-SO Valais), Sierre, Switzerland
}

Hand amputation can dramatically affect the capabilities of a person. Cortical reorganization occurs in the brain, but the motor and somatosensorial cortex can interact with the remnant muscles of the missing hand even many years after the amputation, leading to the possibility to restore the capabilities of hand amputees through myoelectric prostheses. Myoelectric hand prostheses with many degrees of freedom are commercially available and recent advances in rehabilitation robotics suggest that their natural control can be performed in real life. The first commercial products exploiting pattern recognition to recognize the movements have recently been released, however the most common control systems are still usually unnatural and must be learned through long training. Dexterous and naturally controlled robotic prostheses can become reality in the everyday life of amputees but the path still requires many steps.

OPEN ACCESS

Edited by:

Mikhail Lebedev, Duke University, USA

Reviewed by: Ning Jiang,

University of Waterloo, Canada Kianoush Nazarpour, Newcastle University, UK Agamemnon Krasoulis, University of Edinburgh, UK

*Correspondence: Manfredo Atzori manfredo.atzori@hevs.ch

Received: 20 July 2015 Accepted: 09 November 2015 Published: 30 November 2015

Citation:

Atzori M and Müller H (2015) Control Capabilities of Myoelectric Robotic

Prostheses by Hand Amputees: A Scientific Research and Market

Overview.

Front. Syst. Neurosci. 9:162. doi: 10.3389/fnsys.2015.00162
This mini-review aims to improve the situation by giving an overview of the advancements in the commercial and scientific domains in order to outline the current and future chances in this field and to foster the integration between market and scientific research.

Keywords: electromyography, prosthetics, rehabilitation robotics, machine learning

\section{INTRODUCTION}

It is estimated that 41,000 persons were living with a major loss of an upper limb in 2005 (Ziegler-Graham et al., 2008). A hand amputation is one of the most impairing injuries and it can dramatically affect the capabilities of a person. Recent scientific and commercial advances in manmachine interfaces are promising and suggest that dexterous, naturally controlled, proportional and simultaneous robotic prostheses could be reality in the future of amputees. Nevertheless, the outline of the situation in the market and scientific field is complex and the path to naturally controlled prostheses still requires several steps.

Man-machine interfaces have been developed to control hand prostheses via the brain (Lebedev and Nicolelis, 2006), peripheral nerves (Navarro et al., 2005) or the muscles (Cipriani et al., 2011). The first two methods are promising but they usually require invasive procedures to obtain robust performance, thus they are currently applied only in scientific research. The third method (surface electromyography, sEMG) is probably the most widely used both in commercial settings and in scientific research.

Myoelectric hand prostheses with many degrees of freedom and very good mechanical capabilities are now commercially available. However, prosthetics companies target most of their communication efforts to end users. Thus they highlight the practical capabilities 
of the hands, but they usually do not provide information regarding the technical functionalities and specifications of the prostheses that can be exploitable by academic researchers. Previous papers presented some hand prostheses in detail (Belter et al., 2013) but the market changes quickly.

The scientific research field is even more complex and quickly changing. Many papers have been written in scientific research about the natural control of robotic hands by intact and transradial hand amputated subjects. Most of the methods rely on the use of sEMG and of pattern recognition or proportional control algorithms. The first commercial products exploiting pattern recognition to recognize the movements have recently been released. Targeted muscle reinnervation (TMR) can allow the exploitation of these methods even on subjects with above-elbow amputations. Benchmark databases to compare the performance of different methods and setups have been released (Atzori et al., 2014a). However, several steps are still required to obtain proportional, naturally controlled, robust and usable robotic hand prostheses (bionic hands).

Since the market and the scientific field are so complex and changing so quickly, it can be difficult to have a complete overview of them and to remain constantly updated in both fields. This mini-review aims to be a resource for young and experienced researchers in academia and prosthetic companies by providing a synthetic but complete overview of the current level of advancement in the commercial and scientific reality.

\section{MARKET OUTLINE}

A relatively wide choice of devices is available to restore the capabilities of hand amputees by myoelectric robotic prostheses. Such devices are continuously evolving according to technology, scientific research, market needs and user requirements. The devices usually include two main parts: prosthetic hands and control systems.

\section{Prosthetic Hands}

Currently, hand prostheses include cosmetic prostheses, kinematic prostheses and myoelectric prostheses. Cosmetic prostheses offer esthetical and psychological support. Kinematic prostheses also have functional capabilities, since the user can control the opening and closing of a gripper hand through the motion of the shoulder. Myoelectric prosthesis users can control a battery-powered hand through the electrical signal emitted by the remnant muscles, usually located in the forearm.

The continuous improvements in the field and the different targets and aims of the papers published by the companies can make it difficult for researchers to remain updated with the capabilities of available prostheses. For example, Belter et al. (2013) performed a very thorough description of the mechanical properties of prosthetic hands produced by four companies, but in less than 2 years several companies produced new versions or made substantial changes to the products from a mechanical or electronic point of view.
Thus, the market and research achievements often remain disconnected.

Many prosthetic hands are commercially available. However, few have the capability to reproduce many movements. The following selection represents some of the currently most advanced hand prostheses and gives a representation of different companies and approaches: (1) Touch Bionics i-limb Quantum; (2) Otto Bock Michelangelo; (3) Steeper Bebionic v3; and (4) Vincent hand Evolution 2. Table 1 summarizes the most important features that can be useful in a laboratory. The features are grouped into the following four categories: general technical data, dexterity related features, force related features and control related features.

\section{Control Systems}

Usually two or three sEMG electrodes are located in the socket in correspondence to specific muscles (Figure 1). A myoelectric impulse (i.e., an increase in the amplitude of the electrical signal emitted by the muscles) is used to open and close the prosthetic hand. The number of movements can be increased employing specific (e.g., sequential) control strategies. Such control strategies are usually still far from being natural, thus controlling prostheses requires a high level of skill and a training procedure. Control problems contribute to the scarce capabilities and acceptance of sEMG prostheses (Atkins et al., 1996), but they are likely promising for improvements in a near future.

In Table 1 we summarize some of the most important control related features for the considered prosthetic hands including: number of electrodes, movement control type, movement command and particular features of each control system. As can be noticed in Table 1, despite the mechanical characteristics of the prosthesis allowing to reproduce up to 24 hand movements, the control systems rely in most cases on few (1-3) electrodes and on sequential control strategies or on specific movement triggers (in some cases tunable through a mobile app or other strategies). In sequential control strategies, a specific signal (for example, a simultaneous activation of two sEMG electrodes, usually called co-contraction) is used to switch between a set of predefined movements. In movement triggers on the other hand specific patterns of electrode activation are related to specific movements of the prosthesis. The mentioned methods are not natural, in the sense that they do not correspond to the movement that the subject would have thought to do before the amputation. However, they offer robust results, which is one of the main needs in real life.

Several of the considered prostheses include external sources of information as well. In particular, Touch Bionics i-limb Quantum recently introduced gesture control (recorded via gyroscope, accelerometer and magnetometer) and grip chips (that use blue-tooth chips attached to specific objects) to perform movement selection, while Steeper Bebionic exploits finger position encoders to perform falling object prevention. Sometimes research achievements translate to clinical practice too. In 2013 a pattern recognition system similar to the ones described in the scientific literature was made commercially available (http://www.coaptengineering.com/). 
TABLE 1 | Characteristics of the examined prosthetic hands.

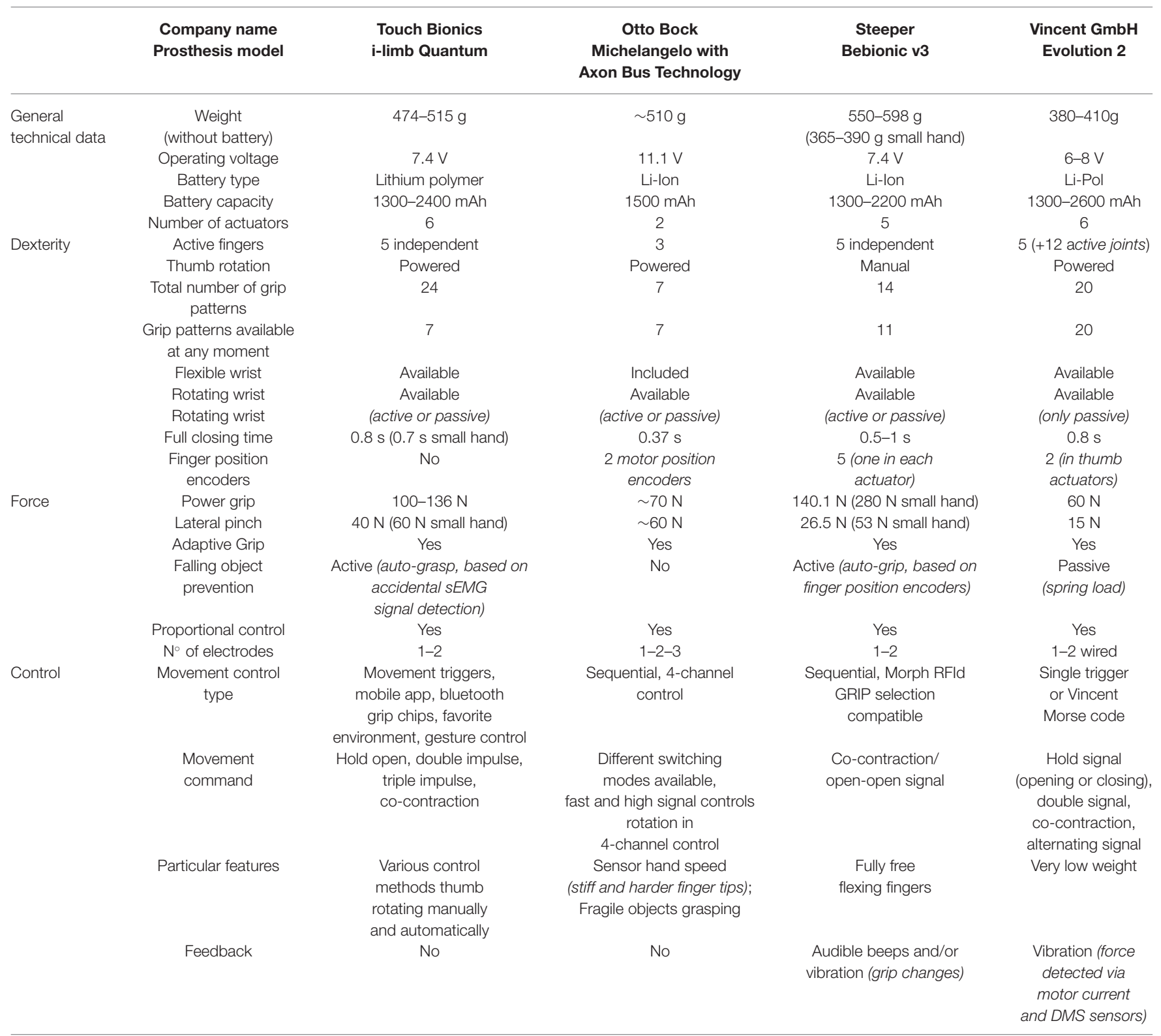

The Coapt system can include up to eight sEMG electrodes. It is generic and it is typically set up to control the number of powered DOFs the patient's prosthesis has. That is, if a powered elbow, wrist, and terminal device are built into the prosthesis then the Coapt system is set to control these. If, however the prosthesis only has a powered terminal device and/or wrist, the Coapt system is set up for those DOFs. Wherever possible, Coapt performs natural control. The technician is encouraged to work with the patient to determine which are the most physiological, repeatable, consistent, and intuitive movements to use for control. Slight variations can be attempted if necessary, also through recalibration procedures. The number of natural grasping patterns that can be achieved varies. According to Coapt, typically users can select between 3-6 naturally. It should be noted that the physical interconnection of the Coapt system and several prostheses has yet to be implemented. An example of movement-triggered control that we received by Coapt is the following one:

1. Hand closing: closing prosthesis.

2. Hand opening: opening prosthesis.

3. Wrist clockwise/counterclockwise rotation: powered wrist clockwise/counterclockwise rotation.

4. Double impulse of natural hand opening: grip A.

5. Triple impulse of natural hand opening: grip B.

6. Holding the hand open: grip C.

7. Single impulse of natural hand opening: grip D. 


\section{SCIENTIFIC RESEARCH OUTLINE}

Many papers have been written in scientific research about the control of robotic hands and prostheses by intact and hand amputated subjects.

Usually several electrodes are placed on the forearm of the subject to record the myoelectric signals (Figure 1) with a dense sampling approach (Fukuda et al., 2003; Tenore et al., 2009; Li et al., 2010) or a precise anatomical positioning strategy (De Luca, 1997; Castellini et al., 2009a). The most common control procedures can be subdivided into pattern recognition or proportional control approaches, which can be applied to sEMG and multimodal signals.

Pattern recognition algorithms are used to classify the movement that the subject aims to perform according to a label (Scheme and Englehart, 2011). Pattern recognition results provided in several cases classification accuracy over $90-95 \%$ on less than 10 classes (e.g., Castellini et al., 2009b), however average results are usually below $80-90 \%$ (Peerdeman et al., 2011). Movement classification methods require movement labeling and they are restricted to a predetermined set of hand movements. Simultaneous pattern recognition has been studied recently (Jiang et al., 2013b; Ortiz-Catalan et al., 2013; Young et al., 2013), however usually such procedures consider simultaneous motions as new classes, thus they can reduce the robustness of the classifier.

Proportional and simultaneous control of a large number of degrees of freedom of the prosthesis can allow achieving more natural and dexterous control using unsupervised or supervised methods (Fougner et al., 2012; Farina et al., 2014). Unsupervised methods are usually based on signal factorization (e.g., through Non-Negative Matrix Factorization, NMF), they require a short calibration phase and they are relatively independent on the

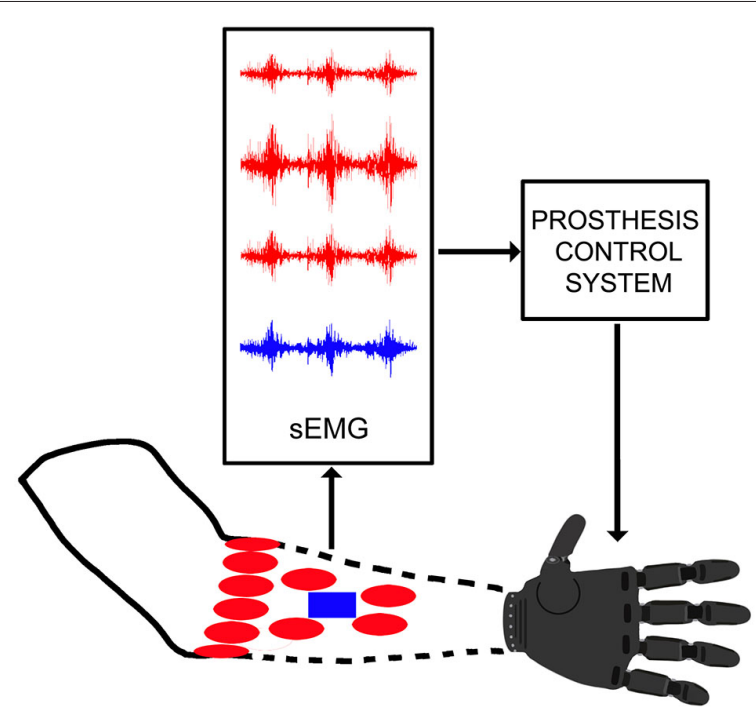

FIGURE 1 | Scheme of a generic myoelectric control system: (i) for commercial prosthesis without pattern recognition (blue rectangle); and (ii) for research (or control system with pattern recognition; red ellipses). The same architecture is assumed in the external forearm. number and exact location of the electrodes (Jiang et al., 2009, 2014a,b; Muceli et al., 2014). Supervised methods (Nielsen et al., 2011; Muceli and Farina, 2012; Ameri et al., 2014a,b; Gijsberts et al., 2014b; Hahne et al., 2014) are usually based on regression techniques (e.g., Linear Regression, LR, Artificial Neural Networks, ANN, Support Vector Machines, SVM) that require a reliable ground truth for hand kinematics. This is easy for intact subjects (e.g., using data gloves), but it can be difficult for amputees, for whom the ground truth can be acquired only via bilateral mirrored contractions (Nielsen et al., 2011) or via visual cues (Ameri et al., 2014a,b). Recently, semisupervised methods (NMF) and supervised methods (LR, ANN) were compared to evaluate the impact of precise kinematics estimation for accurately completing goal-directed tasks (Jiang et al., 2014b). The results showed that, although the three algorithms' mapping accuracies were significantly different, their online performance was similar. These results underline the hypothesis that good proportional myoelectric control can be achieved by the interaction and adaptation of the user with the myoelectric controller through closed-loop feedback. The same hypothesis is also demonstrated in other recent papers on multiple degrees of freedom for intact subjects (Pistohl et al., 2013; Antuvan et al., 2014) and hand amputees (Jiang et al., 2014a). Despite most of the proportional studies concentrating on full hand movements (e.g., hand supination, pronation, rotation, flexion, extension), proportional and simultaneous control has a strong potential for decoding finger kinematics as well. In particular, recent work described average correlation coefficients of up to 0.9 for the estimation of single finger movements (Smith et al., 2008) and 0.8 for the estimation of simultaneous and complex movements (Ngeo et al., 2014).

Also in scientific research, additional sources of information can be used to improve the performance of myoelectric control. Computer vision has been integrated to predetermine the type and size of the required grasp in relation to the object (Došen et al., 2010; Markovic et al., 2014). Accelerometers showed excellent capabilities to recognize hand movements using pattern recognition and regression methods, both alone and in combination with sEMG electrodes (Atzori et al., 2014b; Gijsberts et al., 2014a; Krasoulis et al., 2015).

A common problem in the field is that often the studies are highly specific and they are not directly comparable, due to different acquisition setups, protocols and analysis pipelines. Moreover, often the datasets are not publicly available. The NinaPro project (Atzori et al., 2015) released a publicly available benchmark with EMG, kinematic and dynamic data sources from intact and amputated subjects to help the scientific community to overcome control problems (http://ninaweb.hevs.ch/). Ninapro was recently used to evaluate regression methods for the continuous decoding of finger movements from sEMG and accelerometry (Krasoulis et al., 2015), to apply Dynamic time warping (DTW) in the context of myoelectric control (AbdelMaseeh et al., 2015) and to present the Movement Error Rate, an alternative to the standard window-based accuracy in pattern recognition (Gijsberts et al., 2014a).

Many factors can theoretically influence sEMG controlled prosthesis, including anatomical characteristics of the subjects 
(Farina et al., 2002), training in using myoelectric prostheses (Cipriani et al., 2011), clinical parameters of the subjects (e.g., level of the amputation, phantom limb sensation intensity; Atzori et al., in press), fatigue, sweating, changes in electrode or arm positioning, surgical procedures used during the amputation and even cortical reorganization. However, few studies addressed these effects.

Implanting intramuscular EMG-recording devices reduces the number of parameters affecting the EMG signal and it can improve simultaneous control of multi-DOF prosthetic wrist and hand (Smith et al., 2014, 2015).

TMR is a surgical procedure that redirects the nerves that used to control the muscles of the hand to innervate accessory muscles from which surface sEMG is recorded. Impressive results have been obtained with this method, especially in persons with above-elbow or shoulder amputations (Kuiken et al., 2009). The same technique has also been applied on muscles transferred to the forearm to better integrate with traditional commercial prostheses (Aszmann et al., 2015).

The opposite neural direction, i.e., transferring information from the hand prosthesis to the brain, has been studied in several papers as well. Several attempts have been performed using non-invasive or invasive methods. Electrocutaneous and vibratory stimulation channels have been extensively studied in the past Szeto and Saunders (1982). TMR represents a promising solution also in this case, since it theoretically allows a certain amount of sensory feedback (Marasco et al., 2009). However, to date, the only example of real-time use of neural interfaces for the effective bidirectional control of dexterous prosthetic hands performing different grasping tasks is given by Raspopovic et al. (2014).

Despite the achievements described in this article, there are still several challenges before amputees can benefit from the mentioned signal processing developments (Jiang et al., 2012). First, robustness is probably the most important and challenging problem, in particular for simultaneous and proportional control. Second, the sensory-motor loop should be closed with proper feedback systems, thus opening new possibilities for effective and intuitive prosthetic control. Third, most of the studies are performed in controlled laboratory conditions with non-amputated subjects, which do not adapt to several different real life conditions of amputees (Fougner et al., 2011; Jiang et al., 2013a; He et al., 2015a,b).

\section{CONCLUSIONS}

Hand amputation can dramatically affect the capabilities of a person. The augmentation of the functionalities of the

\section{REFERENCES}

AbdelMaseeh, M., Chen, T.-W., and Stashuk, D. (2015). Extraction and classification of multichannel electromyographic activation trajectories for hand movement recognition. IEEE Trans. Neural Syst. Rehabil. Eng. doi: 10. 1109/tnsre.2015.2447217 [Epub ahead of print]. nervous and muscular system through external devices can already improve the situation of amputees. The market and the scientific field are complex and changing quickly, thus it is often difficult for young researchers to have a complete overview of them, as well as for experienced researchers to remain constantly updated in both the fields. In this mini review, we provide a synthetic but complete overview of the current level of advancement in the commercial and scientific reality, addressing each field in a specific section.

The commercial outline highlights the existence of very advanced prosthetic hands and control systems. Four of the most advanced prosthetic hands were analyzed, showing important mechanical and control differences. In particular, the number of actuators ranges between 2 (Otto Bock Michelangelo), 5 (Steeper Bebionic 3) and 6 (Touch Bionics ilimb Quantum and Vincent Evolution 2) while the number of finger position encoders ranges between 0 (Touch Bionics ilimb Quantum), 2 (Otto Bock Michelangelo, Vincent Evolution 2) and 5 (Steeper Bebionic 3). The first commercial control system based on pattern recognition has been released and it seems a great advancement with respect to previous ones. However natural, proportional and simultaneous control of a large number of degrees of freedom is currently not available.

The scientific research outline shows a large variety of control methods and several possible improvements. Pattern recognition, proportional control and TMR are extremely promising. Common sEMG data resources and benchmarks have been proposed recently to compare different sEMG analysis methods. Most of the factors that can theoretically affect the control of myoelectric prostheses, such as clinical data (e.g., level of the amputation, phantom limb sensation intensity) were recently studied. Finally sensorial feedback recently showed very promising advancements.

In conclusion, the path to proportional, naturally controlled, robust and usable robotic hand prostheses with sensorial feedback (bionic hands) seems to be well initiated and extremely promising for the coming years even though it is still a challenging work in progress.

\section{ACKNOWLEDGMENTS}

We would like to thank Valentina Baruchello and Hugh Gill at Touch Bionics, Bruce Rattray at Steeper, Stefan Schultz at Vincent, Blair Lock at Coapt Engineering, Martin Wehrle, Amsüss Sebastian and Karsten Ley at Otto Bock, and their teams for their helpfulness in providing information for this study.

Ameri, A., Kamavuako, E. N., Scheme, E. J., Englehart, K. B., and Parker, P. A. (2014a). Real-time, simultaneous Myoelectric control using visual target-based training paradigm. Biomed. Signal Process. Control 13, 8-14. doi: 10.1016/j. bspc.2014.03.006

Ameri, A., Kamavuako, E. N., Scheme, E. J., Englehart, K. B., and Parker, P. (2014b). Support vector regression for improved real-time, simultaneous 
Myoelectric control. IEEE Trans. Neural Syst. Rehabil. Eng. 22, 1198-1209. doi: 10.1109/TNSRE.2014.2323576

Antuvan, C. W., Ison, M., and Artemiadis, P. (2014). Embedded human control of robots using Myoelectric interfaces. IEEE Trans. Neural Syst. Rehabil. Eng. 22, 820-827. doi: 10.1109/TNSRE.2014.2302212

Aszmann, O. C., Roche, A. D., Salminger, S., Paternostro-Sluga, T., Herceg, M., Sturma, A., et al. (2015). Bionic reconstruction to restore hand function after brachial plexus injury: a case series of three patients. Lancet 385, 2183-2189. doi: 10.1016/s0140-6736(14)61776-1

Atkins, D. J., Heard, D. C. Y., and Donovan, W. H. (1996). Epidemiologic overview of individuals with upper-limb loss and their reported research priorities. J. Prosthet. Orthot. 8, 2-11. doi: 10.1097/00008526-19960081000003

Atzori, M., Gijsberts, A., Castellini, C., Caputo, B., Hager, A.-G. M., Elsig, S., et al. (2014a). Electromyography data for non-invasive naturally-controlled Robotic hand prostheses. Sci. Data 1:140053. doi: 10.1038/sdata.2014.53

Atzori, M., Gijsberts, A., Müller, H., and Caputo, B. (2014b). Classification of hand movements in amputated subjects by sEMG and accelerometers, in Annual International Conference of the IEEE Engineering in Medicine and Biology Society (EMBC) (Chicago, IL: IEEE), 3545-3549.

Atzori, M., Gijsberts, A., Castellini, C., Caputo, B., Mittaz Hager, A.-G., Elsig, S., et al. (in press). Clinical parameter effect on the capability to control Myoelectric Robotic prosthetic hands. J. Rehabil. Res. Dev. [Epub ahead of print].

Atzori, M., Gijsberts, A., Kuzborskij, I., Elsig, S., Mittaz Hager, A.-G., Deriaz, O., et al. (2015). Characterization of a benchmark database for Myoelectric movement classification. IEEE Trans. Neural Syst. Rehabil. Eng. 23, 73-83. doi: 10.1109/TNSRE.2014.2328495

Belter, J. T., Segil, J. L., Dollar, A. M., and Weir, R. F. (2013). Mechanical design and performance specifications of anthropomorphic prosthetic hands: a review. J. Rehabil. Res. Dev. 50, 599-618. doi: 10.1682/jrrd.2011.10.0188

Castellini, C., Fiorilla, A. E., and Sandini, G. (2009a). Multi-subject/daily-life activity EMG-based control of mechanical hands. J. Neuroeng. Rehabil. 6:41. doi: 10.1186/1743-0003-6-41

Castellini, C., Gruppioni, E., Davalli, A., and Sandini, G. (2009b). Fine detection of grasp force and posture by amputees via surface electromyography. J. Physiol. Paris 103, 255-262. doi: 10.1016/j.jphysparis.2009.08.008

Cipriani, C., Antfolk, C., Controzzi, M., Lundborg, G., Rosen, B., Carrozza, M. C., et al. (2011). Online Myoelectric control of a dexterous hand prosthesis by transradial amputees. IEEE Trans. Neural Syst. Rehabil. Eng. 19, 260-270. doi: 10.1109/TNSRE.2011.2108667

De Luca, C. J. (1997). The use of surface electromyography in biomechanics. J. Appl. Biomech. 13, 135-163.

Došen, S., Cipriani, C., Kostić, M., Controzzi, M., Carrozza, M. C., and Popović, D. B. (2010). Cognitive vision system for control of dexterous prosthetic hands: experimental evaluation. J. Neuroeng. Rehabil. 7:42. doi: 10.1186/17430003-7-42

Farina, D., Cescon, C., and Merletti, R. (2002). Influence of anatomical, physical and detection-system parameters on surface EMG. Biol. Cybern. 86, 445-456. doi: 10.1007/s00422-002-0309-2

Farina, D., Jiang, N., Rehbaum, H., Holobar, A., Graimann, B., Dietl, H., et al. (2014). The extraction of neural information from the surface EMG for the control of upper-limb prostheses: emerging avenues and challenges. IEEE Trans. Neural Syst. Rehabil. Eng. 22, 797-809. doi: 10.1109/TNSRE.2014. 2305111

Fougner, A., Scheme, E., Chan, A. D. C., Englehart, K., and Stavdahl, O. (2011). Resolving the limb position effect in Myoelectric pattern recognition. IEEE Trans. Neural Syst. Rehabil. Eng. 19, 644-651. doi: 10.1109/TNSRE.2011. 2163529

Fougner, A., Stavdahl, Ø., Kyberd, P. J., Losier, Y. G., and Parker, P. A. (2012). Control of upper limb prostheses: terminology and proportional Myoelectric control-a review. IEEE Trans. Neural Syst. Rehabil. Eng. 20, 663-677. doi: 10. 1109/TNSRE.2012.2196711

Fukuda, O., Tsuji, T., Kaneko, M., Otsuka, A., and Tsuji, O. F. T. (2003). A humanassisting manipulator teleoperated by EMG signals and arm motions. IEEE Trans. Robot. Autom. 19, 210-222. doi: 10.1109/tra.2003.808873

Gijsberts, A., Atzori, M., Castellini, C., Muller, H., and Caputo, B. (2014a). The movement error rate for evaluation of machine learning methods for sEMG- based hand movement classification. IEEE Trans. Neural Syst. Rehabil. Eng. 22, 735-744. doi: 10.1109/TNSRE.2014.2303394

Gijsberts, A., Bohra, R., González, D. S., Werner, A., Nowak, M., Caputo, B., et al. (2014b). Stable Myoelectric control of a hand prosthesis using non-linear incremental learning. Front. Neurorobot. 8:8. doi: 10.3389/fnbot.2014.00008

Hahne, J. M., BieBmann, F., Jiang, N., Rehbaum, H., Farina, D., Meinecke, F. C., et al. (2014). Linear and nonlinear regression techniques for simultaneous and proportional Myoelectric control. IEEE Trans. Neural Syst. Rehabil. Eng. 22, 269-279. doi: 10.1109/TNSRE.2014.2305520

He, J., Zhang, D., Jiang, N., Sheng, X., Farina, D., and Zhu, X. (2015a). User adaptation in long-term, open-loop Myoelectric training: implications for EMG pattern recognition in prosthesis control. J. Neural Eng. 12:046005. doi: $10.1088 / 1741-2560 / 12 / 4 / 046005$

He, J., Zhang, D., Sheng, X., Li, S., and Zhu, X. (2015b). Invariant surface EMG feature against varying contraction level for Myoelectric control based on muscle coordination. IEEE J. Biomed. Health Inform. 19, 874-882. doi: 10. 1109/JBHI.2014.2330356

Jiang, N., Dosen, S., Muller, K., and Farina, D. (2012). Myoelectric control of artificial limbs-is there a need to change focus? IEEE Signal Process. Mag. 29, 150-152. doi: 10.1109/msp.2012.2203480

Jiang, N., Englehart, K. B., Parker, P. A., and Englehart, K. B. (2009). Extracting simultaneous and proportional neural control information for multiple degree of freedom prostheses from the surface electromyographic signal. IEEE Trans. Biomed. Eng. 56, 1070-1080. doi: 10.1109/TBME.2008.2007967

Jiang, N., Muceli, S., Graimann, B., and Farina, D. (2013a). Effect of arm position on the prediction of kinematics from EMG in amputees. Med. Biol. Eng. Comput. 51, 143-151. doi: 10.1007/s11517-012-0979-4

Jiang, N., Tian, L., Fang, P., Dai, Y., and Li, G. (2013b). Motion recognition for simultaneous control of multifunctional transradial prostheses, in Engineering in Medicine and Biology Society (EMBC), 2013 35th Annual International Conference of the IEEE (Osaka: IEEE), 1603-1606.

Jiang, N., Rehbaum, H., Vujaklija, I., Graimann, B., and Farina, D. (2014a). Intuitive, online, simultaneous and proportional Myoelectric control over two degrees-of-freedom in upper limb amputees. IEEE Trans. Neural Syst. Rehabil. Eng. 22, 501-510. doi: 10.1109/TNSRE.2013.2278411

Jiang, N., Vujaklija, I., Rehbaum, H., Graimann, B., and Farina, D. (2014b). Is accurate mapping of EMG signals on kinematics needed for precise online Myoelectric control? IEEE Trans. Neural Syst. Rehabil. Eng. 22, 549-558. doi: $10.1109 /$ tnsre.2013.2287383

Krasoulis, A., Vijayakumar, S., and Nazarpour, K. (2015). Evaluation of regression methods for the continuous decoding of finger movement from surface EMG and accelerometry, in Neural Engineering (NER), 2015 7th International IEEE/EMBS Conference on (Montpellier: IEEE), 631-634.

Kuiken, T. A., Li, G., Lock, B. A., Lipschutz, R. D., Miller, L. A., Stubblefield, K. A., et al. (2009). Targeted muscle reinnervation for real-time Myoelectric control of multifunction artificial arms. JAMA 301, 619-628. doi: 10.1001/jama. 2009.116

Lebedev, M. A., and Nicolelis, M. A. L. (2006). Brain-machine interfaces: past, present and future. Trends Neurosci. 29, 536-546. doi: 10.1016/j.tins. 2006.07.004

Li, G., Schultz, A. E., and Kuiken, T. A. (2010). Quantifying pattern recognitionbased Myoelectric control of multifunctional transradial prostheses. IEEE Trans. Neural Syst. Rehabil. Eng. 18, 185-192. doi: 10.1109/TNSRE.2009. 2039619

Marasco, P. D., Schultz, A. E., and Kuiken, T. A. (2009). Sensory capacity of reinnervated skin after redirection of amputated upper limb nerves to the chest. Brain 132, 1441-1448. doi: 10.1093/brain/awp082

Markovic, M., Dosen, S., Cipriani, C., Popovic, D., and Farina, D. (2014). Stereovision and augmented reality for closed-loop control of grasping in hand prostheses. J. Neural Eng. 11:046001. doi: 10.1088/1741-2560/11/4/046001

Muceli, S., and Farina, D. (2012). Simultaneous and proportional estimation of hand kinematics from EMG during mirrored movements at multiple degreesof-freedom. IEEE Trans. Neural Syst. Rehabil. Eng. 20, 371-378. doi: 10. 1109/TNSRE.2011.2178039

Muceli, S., Jiang, N., and Farina, D. (2014). Extracting signals robust to electrode number and shift for online simultaneous and proportional Myoelectric control by factorization algorithms. IEEE Trans. Neural Syst. Rehabil. Eng. 22, 623-633. doi: 10.1109/TNSRE.2013.2282898 
Navarro, X., Krueger, T. B., Lago, N., Micera, S., Stieglitz, T., and Dario, P. (2005). A critical review of interfaces with the peripheral nervous system for the control of neuroprostheses and hybrid bionic systems. J. Peripher. Nerv. Syst. 10, 229-258. doi: 10.1111/j.1085-9489.2005.10303.x

Ngeo, J. G., Tamei, T., and Shibata, T. (2014). Continuous and simultaneous estimation of finger kinematics using inputs from an EMG-to-muscle activation model. J. Neuroeng. Rehabil. 11:122. doi: 10.1186/1743-0003-11-122

Nielsen, J. L. G., Holmgaard, S., Jiang, N., Englehart, K. B., Farina, D., and Parker, P. A. (2011). Simultaneous and proportional force estimation for multifunction Myoelectric prostheses using mirrored bilateral training. IEEE Trans. Biomed. Eng. 58, 681-688. doi: 10.1109/TBME.2010.2068298

Ortiz-Catalan, M., Branemark, R., and Hakansson, B. (2013). Evaluation of classifier topologies for the real-time classification of simultaneous limb motions. Conf. Proc. IEEE Eng. Med. Biol. Soc. 2013, 6651-6654. doi: 10. 1109/EMBC.2013.6611081

Peerdeman, B., Boere, D., Witteveen, H., Huis in 't Veld, R., Hermens, H., Stramigioli, S., et al. (2011). Myoelectric forearm prostheses: state of the art from a user-centered perspective. J. Rehabil. Res. Dev. 48, 719-738. doi: 10. 1682/jrrd.2010.08.0161

Pistohl, T., Cipriani, C., Jackson, A., and Nazarpour, K. (2013). Abstract and proportional Myoelectric control for multi-fingered hand prostheses. Ann. Biomed. Eng. 41, 2687-2698. doi: 10.1007/s10439-013-0876-5

Raspopovic, S., Capogrosso, M., Petrini, F. M., Bonizzato, M., Rigosa, J., Di Pino, G., et al. (2014). Restoring natural sensory feedback in real-time bidirectional hand prostheses. Sci. Transl. Med. 6:222ra19. doi: 10.1126/scitranslmed. 3006820

Scheme, E., and Englehart, K. (2011). Electromyogram pattern recognition for control of powered upper-limb prostheses: state of the art and challenges for clinical use. J. Rehabil. Res. Dev. 48, 643-659. doi: 10.1682/jrrd.2010. 09.0177

Smith, L. H., Kuiken, T. A., and Hargrove, L. J. (2014). Real-time simultaneous and proportional Myoelectric control using intramuscular EMG. J. Neural Eng. 11:066013. doi: 10.1088/1741-2560/11/6/066013
Smith, L. H., Kuiken, T. A., and Hargrove, L. J. (2015). Linear regression using intramuscular EMG for simultaneous Myoelectric control of a wrist and hand system, in Neural Engineering (NER), 2015 7th International IEEE/EMBS Conference on (Montpellier: IEEE), 619-622.

Smith, R. J., Tenore, F., Huberdeau, D., Cummings, R. E., and Thakor, N. V. (2008). Continuous decoding of finger position from surface EMG signals for the control of powered prostheses. Conf. Proc. IEEE Eng. Med. Biol. Soc. 2008, 197-200. doi: 10.1109/IEMBS.2008.4649124

Szeto, A. Y. J., and Saunders, F. A. (1982). Electrocutaneous stimulation for sensory communication in rehabilitation engineering. IEEE Trans. Biomed. Eng. 4, 300-308. doi: 10.1109/tbme.1982.324948

Tenore, F. V. G., Ramos, A., Fahmy, A., Acharya, S., Etienne-Cummings, R., and Thakor, N. V. (2009). Decoding of individuated finger movements using surface electromyography. IEEE Trans. Biomed. Eng. 56, 1427-1434. doi: 10. 1109/TBME.2008.2005485

Young, A. J., Smith, L. H., Rouse, E. J., and Hargrove, L. J. (2013). Classification of simultaneous movements using surface EMG pattern recognition. IEEE Trans. Biomed. Eng. 60, 1250-1258. doi: 10.1109/TBME.2012.2232293

Ziegler-Graham, K., MacKenzie, E. J., Ephraim, P. L., Travison, T. G., and Brookmeyer, R. (2008). Estimating the prevalence of limb loss in the United States: 2005 to 2050. Arch. Phys. Med. Rehabil. 89, 422-429. doi: 10.1016/j. apmr.2007.11.005

Conflict of Interest Statement: The authors declare that the research was conducted in the absence of any commercial or financial relationships that could be construed as a potential conflict of interest.

Copyright (c) 2015 Atzori and Müller. This is an open-access article distributed under the terms of the Creative Commons Attribution License (CC BY). The use, distribution and reproduction in other forums is permitted, provided the original author(s) or licensor are credited and that the original publication in this journal is cited, in accordance with accepted academic practice. No use, distribution or reproduction is permitted which does not comply with these terms. 